\title{
The Design and Application of Service Project Management System Based on ODPS Platform
}

\author{
Wentao Zhu \& Qiang Yu \\ Department of Management, Hebei University, Baoding, Hebei, China
}

Jianli Yang

Department of Electronic and Information Engineering, Hebei University, Baoding, Hebei, China

zhuwentao00@126.com,yuqiang@hbu.edu.cn,yangjianli_1987@126.com

\begin{abstract}
Because of ineffective management, it appears schedule delay, materials supply not in time, cost overruns, human resource management confusion, security management and quality management activities carried out in disorder during the process of wind turbines service projects at this time. The paper designs an advanced service project management system based on ODPS platform which can be integrated with other systems in order to standardize the management of wind turbines service project management, realize the scientific process of information and automation, and enhance the competitiveness of wind turbines service enterprises.
\end{abstract}

KEYWORD: ODPS Platform; Wind Turbine; Service Project Management System

\section{INTRODUCTION}

\subsection{Current problems}

As the reducing of the fossil energy, the living environment is going worse, clean energy and renewable energy are taken more and more attention. In the process, wind power has been developed as the fourth power energy source after thermal power, hydropower and nuclear power, in developed countries. The daily work of wind turbines service enterprises is mainly around the service projects management. The project schedule, staff efficiency and cost control are directly related to the enterprise operation level. The enterprise exists or not is depended on what kind of information system used by service project management.

\subsection{The current situation of the project management system development}

Microsoft Office Project supports simple graphics and images which can suit general projects in many areas. But the use of it seriously limited in the mobile internet era. Based on the $\mathrm{B} / \mathrm{S}$ structure, Oracle Primavera P6 created by Primavera System inc integrates project plan, schedule and resource, used in many large or mega construction projects. But because of versatility and limitedness of project schedule and resource management, it shows lack of functional requirements in the enterprise operation management.

\subsection{Purposes}

Base on the actual investigation of service project management mode and business process in the wind turbines service corporation, and using of ODPS platform, to design a service project management which can realize the information, automation standardization and reflect enterprise operation status constantly. And it can provide basis for decision making through the multi-information management system platform.

\section{THE FRAMEWORK OF THE SYSTEM}

\subsection{The whole description}

The system designed in this paper is based on the data of projects, staff and material inventory. The system regards service projects as the core; regards services management, the support for decisions and services to staff as specific targets. The service project management system integrates human resource management, project management, warehouse management, technical documents and data management, information sharing platform as a unit. It is a highly collaborative system, business management information system and intelligent decision system. Through the rapid exchange and integration of various technologies and business data, so as to achieve projects management, projects business regulation and other business automation and standardization, to promote management tools and methods; to integrate with OA, ORACLE ERP, 
financial and other systems.

\subsection{Association framework}

Through the transmission medium such as optical fiber, cable networks, wireless networks to construct a comprehensive project management system, which regards projects management as core business, project tasks as main line and connects the relevant business. It can be accessed by computers, smart phones and other devices. System associated frame schematic is shown below:

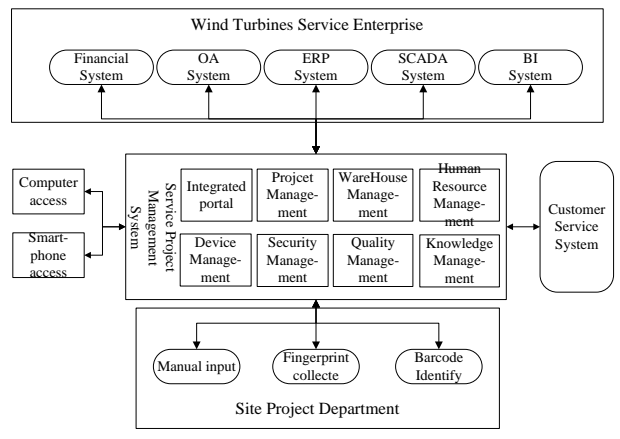

Figure -1 Associated framework diagram
Service project management system is a self management system and closely relates with the financial system, the OA office automation system, ERP system, SCADA system, BI system and other systems. It's the core business management system for wind turbines service enterprises.

\subsection{System design}

This system adopts "5-layers\&3-system" design pattern. 5 layers are IT infrastructure layer, presentation layer, application layer, application support layer and information resource layer. 3 systems are information standardization system, security system and operation management system. In order to realize the construction scheduled targets, using the design idea of platform and modular; relying on unified data exchange, unified interface standard, and unified security uniform. As shown below:

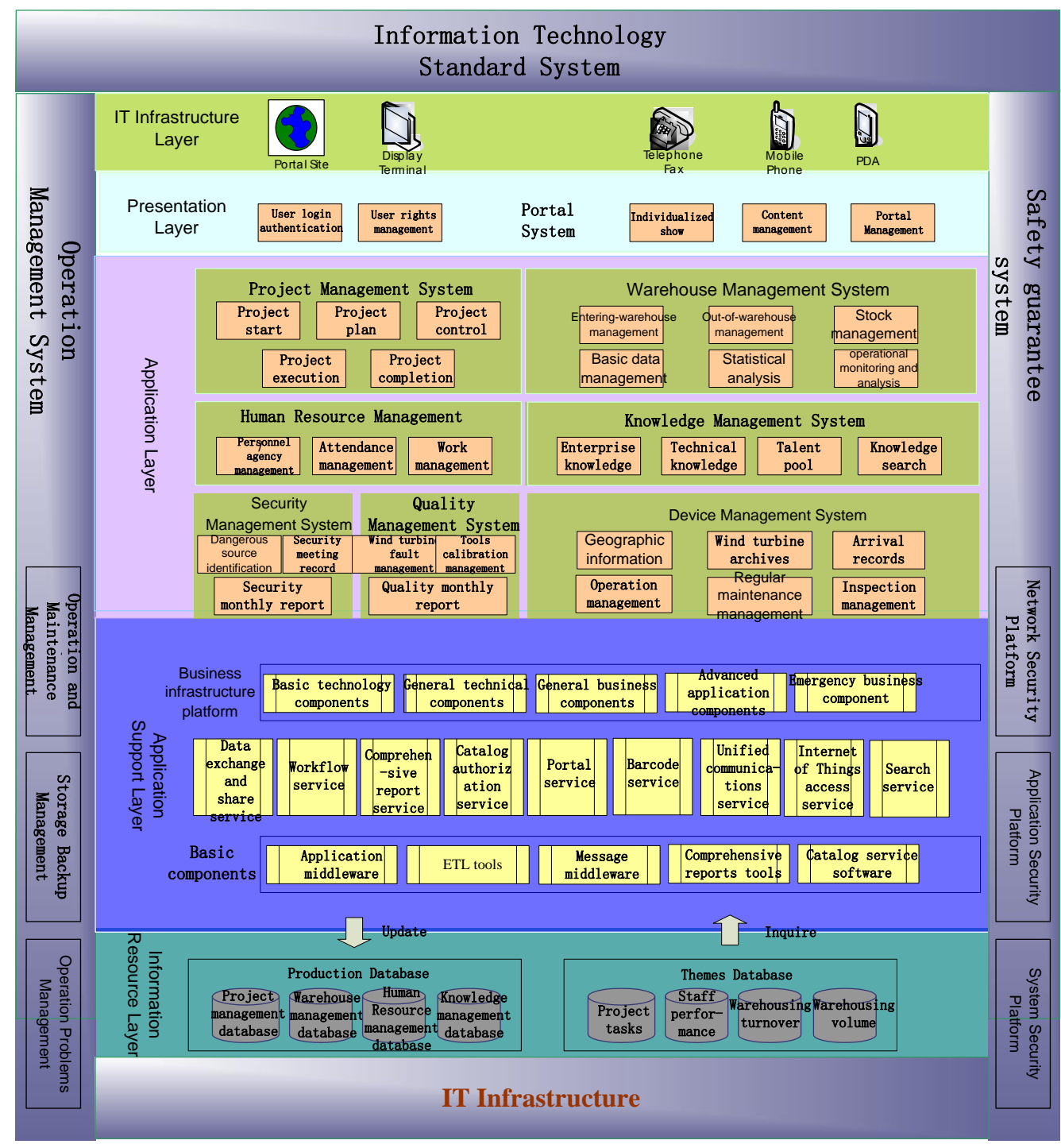

Figure -2 Service project management system design pattern diagram 


\subsubsection{The description of system hierarchy design}

IT Infrastructure Layer provides the basis environment for system operation, including three parts, network communication, host storage, and system software. The network section includes VPN, internet and so on. Host storage section includes host, storage, backup recovery and other integrated facilities. System software includes operating systems, database software.

Presentation layer is responsible for interacting with the user, accepting service requests, integrating application, sharing resource retrieval, publishing information, and other functions. The content in this layer is shown in the form of a comprehensive portal, through the browser usually. Visitors can log one time into multiple systems, which is flexible business processes and integration, through computer, PDA, or cell phone. This layer can realize flexible collocation and integration of business processes.

Application layer is based on framework, platform, services and tools supported by application support layer. Database access layer provides its data resources. Application layer build business applications for all kinds of users of the system, such as project management, human resource management, warehouse management, security management, quality management, device management, knowledge management, portal systems and so on.

Application support layer is a development architecture built over the under layer hardware and software, with componentized software platform; in order to achieve seamless integration of systems management and business applications, to realize business applications developed customized and managed configurable. The coding of business development is reduced greatly. The flexibility and reusability of system application is increasing. Application support layer Includes workflows, rights management, resource directory services, report management, messaging services, system maintenance and other components. It can provide support for the upper application system.

Information resource layer is mainly all kinds of business information processing. Information resource library, total of four databases, includes warehouse database, project management database, human resource management database, knowledge database.

\subsubsection{Support systems description}

To ensure the system can be used widely and operating stable only depends on application development and network construction is not enough. It also needs common supports from three different sides: security, standardization, operation and maintenance. Support system includes three parts. They are information technology standards system, safety guarantee system, operation management system

Information technology standard system is referring to advanced experience of the implementation of e-government and regarding its national standard system as framework. Information technology standard system includes appropriate national and international standards, operation and maintenance debugging norms, standards of computer technology, communications and networking technologies standards. The most important thing is combination with the standard specifications of information systems in use.

Safety guarantee system consists of network security platform, application security platform and system security platform. Network security platform provides security controls of access to information, trust services, basic security protections, failback and disaster recovery services, etc. Application security platform including authentication, access control, data security, system logs, mainly to provide a unified user management services, namely, provide a unified user management, role management, authorization, certification, etc. System security platform includes the operating system security, server security, anti-virus system and so on.

Operation management system includes operation and maintenance management, storage backup management, operation problems management. It means operation, maintenance, support and training system, run through the whole process of the system from designing to running, providing a good operation and maintenance support functions and improving the real-time dynamic documents services.

\section{FUNCTION REALIZATION}

\subsection{The description of ODPS development platform}

The service project management system designed in this paper is entirely based on the ODPS platform. ODPS named Open Document Processing System is an operational framework with advanced concept through abstraction of business functions in numerous projects practice. It's also a technical framework, coming from techniques accumulation, which has developed numerous products. ODPS includes workflow technology, form processors, document processors, document type systems, and other related development tools.

ODPS running framework of application support platform is divided into four layers, they are: presentation layer, request service layer, business layer and resource access layer.

Presentation layer framework designed in full compliance with the MVC model. The controller is used to separate model and view, to achieve the 
effect of loose coupling between layers; and to increase flexibility, reusability and maintainability. In the Web framework, we mainly use JSP, JavaBeans, Taglib, XML and Servlet technologies, etc in J2EE, combined with a variety of classic design mode, the main Web application development technology integrated into a unified framework. Developers do not have to use their own coding to achieve a full development of its MVC mode, which greatly saves time.

Request service layer playing pivot roles, it is responsible for connecting the presentation layer and business layer, and is responsible for converting data between two levels, transferring data and so on. This layer includes business delegate, facade, and data transfer object modules; and is completely transparent to the system developers. In other words, developers do not need to focus on how the client's data package, but also do not care about how to access the business logic layer. They only need to master the methods of how to call business objects needed by business domain layer on request service layer.

Business layer is mainly composed of process controls, filters, business proxy objects, business objects, preservation state, resource control and transaction control module. For developers, they need to develop filter and business object, can also selectively use business proxy object. For the unrelated business logic operation of resource management, transaction management and routing control, developers need only a simple configuration via the configuration file without writing a line of code. The system will take on the management of affairs and resources automatically according to the configuration files written by developers.

For resource access layer, in order to separate persistent storage and business logic completely, the base running framework adopted standard model of DAO. Resource access layer also provides a variety of data access layer DAO implementations, including databases, LDAP, and other file systems. So that developers can stay focus on business logic while developing, without the need of considering the database and LDAP structure.

ODPS platform is based on open standards J2EE technology route, cross-platform applications, insisting loosely coupled design principles, using a large number of middleware and AJXA technologies. While using the CA identity authentication technology, rights management, configuration services and a variety of applications security services, to protect the security of application system.

\subsection{Function Modules}

Service project system overall functions design as shown below:

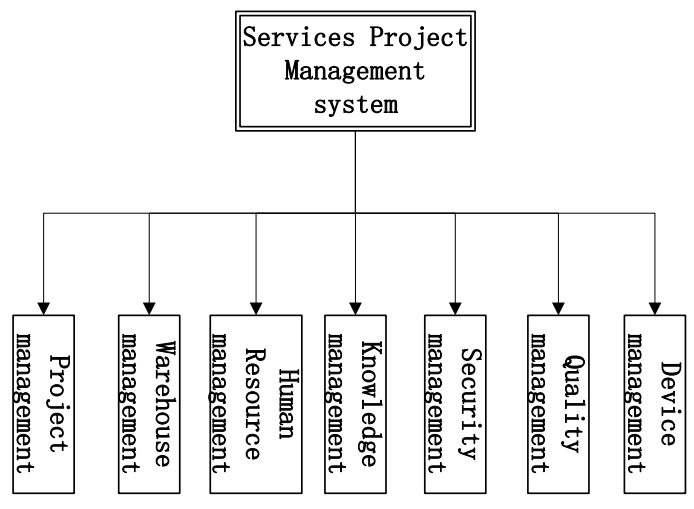

Figure -3 Functions design diagram

Project Management: Under the limited conditions, using the systems integration methods to manage all of the work involved effectively. Mainly contents 5 processes, project planning process, project control process, project execution process, project closure process.

Warehouse Management: The system satisfied the needs of business warehouse management from production, support multi-party system information exchange, easy to expand value-added services, support multi-warehouse and multi-project management. It is mainly divided into 6 parts: basic data management, entering-warehouse management, out-of-warehouse management, stock management, statistical analysis, operational monitoring and analysis.

Human Resource Management: the system can achieve the personnel fingerprint attendance, travel tracking, position management, file management, allowance calculation, human resource planning and deployment, work analysis and other functions.

Knowledge Management: The system establishing enterprise knowledge documentation center and customer service knowledge base. It can realize knowledge planning, knowledge tracking, knowledge map, mutual help knowledge, knowledge evaluation and so on. At the same time, supports documentation sub-libraries, assign permissions, document encryption, publishing approval process, supports standard document formats and Office online editing, and other functions.

Device Management: The system provides the amount of stock decision for the storage manager, through records of each wind turbine parts information, geographic information, delivery time. Through the docking of SCADA system owned by each turbine, constantly monitor all wind power turbines operation state, the key parts operation state and generating capacity, to achieve wind turbines' scientific management of daily inspection and maintenance, regular maintenance of the key parts.

Security Management: All project departments can record dangerous source in the system, and share it to the whole company. We can record in the system such as project security basic information, the implementation of security measures, inspection 
and rectification, violations and penalties, regular safety training and implementation, collection and disposal of wastes, suggestions and summary information. Company level monthly reports will be automatically produced for managers' decisions, through pooled analysis of all of this information via the system.

Quality management: The system realizes functions of inspection records of important tools and measuring tools, and automatically alerts the operators the next inspection date. The system records failure information of wind turbines, such as, time, location, cause, treatment methods, the responsible person, damaged parts and other information. It can generate fault history records, and the automatic identify batches problems after pooled analysis. We can analysis and evaluate the effect of quality work monthly completed after comparison with annual quality objectives pre-set through the system.

\subsection{System deployment}

The overall operating environment of integrated application software mainly involves three types of servers: database server, integrated application server, file server. Specifically, as shown below:

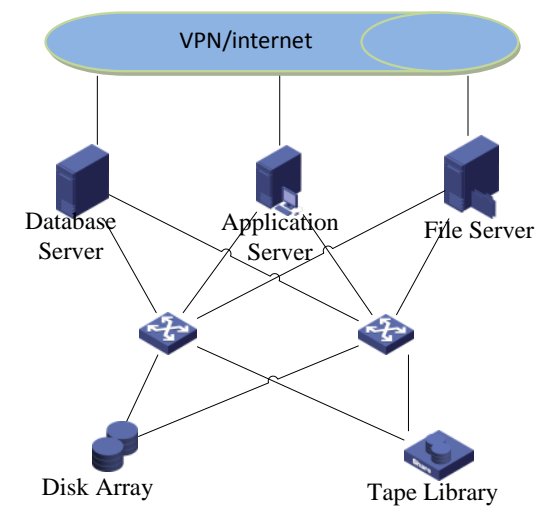

Figure -4 System deployment diagram

\section{CONCLUSION}

This paper analyzes characteristics of wind power turbines service industry business; using ODPS development platform, completes the design and development work for wind power turbines the service project management system. This system has made outstanding contributions in the management decision, leading the innovation management mode, and enhancing enterprises competitiveness. After promoting the use of certain enterprises shows that the system has a strong practical able to realize flexible customization and modifications many elements of project management, which can be carried out with other management information systems convergence and effective transfer and sharing of data, the system fully functional, cover all stages of project management. Enterprises make the system can concentrate business, standardize management, improve efficiency, and at the same time reduce office costs.

\section{REFERENCES}

[1] Kongdecai. "Design and Implementation of Project Management System Based on Critical Chain Technology". Enterprise Management. ShenYangLigong University. 2012.

[2] Wangyong,Zhangbin. PMBOK Guide (Version 4). Publishing House of Electronics Industry.2009.

[3] Liuxuejin, Yuzhiwei. "The project management method in the application of scientific research project management". Science and technology information. 2009 NO.24.

[4] Qinanbang. Project management: a new perspective of management (Version 7). China Renmin University Press. 2011.

[5] Sunjian."Design and Implementation of the Project Management System". Department of Computer Science and Technology, Jilin University, 2013. 